\title{
Blood Pressure and Lipid Profile in Hypertensive Patients Post the First COVID-19 Lockdown: "Brief Letter for Publication"
}

\author{
Ilaria Fucile ${ }^{1} \cdot$ Maria Virginia Manzi $^{1} \cdot$ Costantino Mancusi $^{1}[$
}

Received: 25 June 2021 / Accepted: 6 August 2021 / Published online: 18 August 2021

(C) Italian Society of Hypertension 2021

At the beginning of March 2020, due to daily increasing cases and deaths related to COVID-19 infection, the Italian Government decided for total lockdown. The people had to stay home limiting their movements to strictly essential activities including food shopping, health care and work, when teleworking was not possible. Individual sport activity was not allowed. Schools and universities as well as all non-essential public places, including shops (except for food shopping), restaurants, cafés, cinemas, gym were closed. For this reason the consumption of fresh foods, especially fruit, vegetables and fish reduced, in favor of frozen and/or canned foods. The time spent seated on an average day increased.

All of these changes could adversely affect the control of chronic disease.

Adult patients affected by hypertension may be considered a vulnerable population, since hypertension and diabetes are known risk factors for severe COVID-19-related pneumonia.

Hypertension represents the most important modifiable risk factor for coronary heart disease, stroke, heart failure and kidney failure. Appropriate lifestyle contribute to blood pressure (BP) reduction in hypertensive individuals already on medical therapy. The salt restriction, moderation of alcohol consumption, high consumption of vegetables and fruits and low-fat, weight reduction and maintenance and regular physical exercise have been shown to be capable of reducing BP [1].

The aim of this study was to examine the impact of first lockdown on BP control, weight and lipid profile in hypertensive patients.

We recruited 72 hypertensive patients, 23 women, followed at Hypertension Research Center of Federico II

Costantino Mancusi

costantino.mancusi@unina.it

1 Hypertension Research Center and Department of Advanced Biomedical Science, Federico II University Hospital, Naples, Italy
University Hospital in Naples as part of the Campania Salute Network Registry. The enrolment started at the end of the first lockdown when nationally-imposed restrictions for nonurgent medical activity were removed (May 4th, 2020) until July 2020. We collected body weight, lipid profile, fasting blood glucose and office BP.

From the registry database the same information at their last visit before the lockdown were taken as comparison. No significant changes in body weight, lipid profile and blood glucose were found compared to previous visits while a significant reduction in systolic $(139 \pm 17.55$ vs $132 \pm 20.45)$ and diastolic (78 \pm 9.59 vs $75 \pm 11.57)$ BP was found ( $<<$ $0.05)$.

No patients report withdrawal of antihypertensive therapy during lockdown.

The recent research letter by Pengo et al. analyzed the impact of COVID-19 lockdown on home blood pressure (BP) in hypertensive patients. They showed that during COVID-19 outbreak BP values of 126 enrolled patients significant decreased compared to the corresponding time window of the previous year [2].

Tsigkas et al., through a telephone interview survey of a random sample of 1014 Greek citizens, demonstrated reduction of passive smoking, working hours, alcohol, junk food and salt consumption, and an increase in sleeping hours mainly in participants with a lower burden of cardiovascular risk factors [3]. They suggested that lifestyle changes induced by lockdown measure could reduce the chance for an acute plaque rupture and explain the reduction of acute coronary syndrome incidence. All the reported change exert also a significant effect of BP values.

Similarly, the Italian study of 3533 adults showed that during lockdown smoking habits have been reduced, sleep hours have increased and a higher frequency of training was reported when compared to the previous period [4]. In addition significant improvement on dietary habits was found with an increase in homemade recipes (e.g. sweets, pizza and bread), cereals, legumes, white meat and hot beverages 
consumption, and a decrease in packaging sweets and baked products, delivery food and alcoholics intake. On the other hand, the perception of weight gain was observed in $48.6 \%$ of the population and resulted to be present in people who started the smart working.

Many of these lifestyle habits that were changed favorably during quarantine are well-known modifiable risk factors for hypertension and might be associated with the observed reduction of BP. It is noteworthy to underline that no patients withdrawal the use of any antihypertensive medication following the unjustified alarm on the use of ACE inhibitors during the first phase of COVID-19 pandemic [5].

\section{References}

1. Williams B, Mancia G, Spiering W, AgabitiRosei E, Azizi M, Burnier M. 2018 ESC/ESH Guidelines for the management of arterial hypertension: The Task Force for the management of arterial hypertension of the European Society of Cardiology and the European Society of Hypertension: The Task Force for the management of arterial h. J Hypertens. 2018;36(10):1953-2041.

2. Pengo MF, Albini F, Guglielmi G, Mollica C, Soranna D, Zambra G, Zambon A, Bilo G, Parati G. Home blood pressure during COVID-19-related lockdown in patients with hypertension. Eur J Prev Cardiol. 2021:zwab010. https://doi.org/10.1093/eurjpc/ zwab010.

3. Tsigkas G, Koufou EE, Katsanos K, Patrinos P, Moulias A, Miliordos I, Almpanis G, Christodoulou I, Papanikolaou F, Dimitroula T, Kivetos A, Vardas P, Davlouros P. Potential relationship between lifestyle changes and incidence of hospital admissions for acute coronary syndrome during the COVID-19 lockdown. Front Cardiovasc Med. 2021;8:604374.

4. Di Renzo L, Gualtieri P, Pivari F, Soldati L, Attinà A, Cinelli G, Leggeri C, Caparello G, Barrea L, Scerbo F, Esposito E, De Lorenzo A. Eating habits and lifestyle changes during COVID-19 lockdown: an Italian survey. J Transl Med. 2020;18(1):229.

5. Iaccarino G, Borghi C, Cicero AFG, Ferri C, Minuz P, Muiesan ML, Mulatero P, Mulè G, Pucci G, Salvetti M, Savoia C, Sechi LA, Volpe M, Grassi G. Renin-angiotensin system inhibition in cardiovascular patients at the time of COVID19: much ado for nothing? A statement of activity from the Directors of the Board and the Scientific Directors of the Italian Society of Hypertension. High Blood Press Cardiovasc Prev. 2020;27(2):105-8. 